as Baltimore and London. He also describes the instruments suitable for use in psychiatric screening-questionnaires long and short and interviews standardized or not-and stresses the need for such instruments to be valid, reliable, and stable. In an important section of his paper the author describes a psychiatric screening survey which formed part of a multiple screening programme carried out in one London borough. He achieved only a $70 \%$ acceptance rate, which must limit any conclusions that can be drawn. The screening test used was a selection of 20 items from the Cornell Medical Index. People scoring more than 5 were given a standardized clinical interview. There were $19 \%$ false negatives and $6 \%$ false positives. Moreover, interesting differences appeared between the assessments of the research psychiatrist and of five general practitioners taking part in the project.

In psychiatry the major research effort so far has been directed to psychoses, the severe neuroses, and alcoholism. Eastwood has made a case for devoting more attention to the minor psychiatric disorders and offers some suggestions on how that might be done. Even so, there is no dissenting from his conclusion that "for the present, as with some other forms of morbidity, screening for psychiatric disorder must remain at the experimental phase and is not ready for inclusion in the medical services."

\footnotetext{
${ }^{1}$ Report of the War Office Committee of Enquiry into 'Shell Shock', Cmnd.

1734 . London, H.M.S.O., 1922 .
2 The Work of Psychologists and Psychiatrists in the Services-Report of an Expert Committee. London, H.M.S.O., 1947.

3 Wilson, J. M. G., and Jungner, G., Principles and Practice of Screening for Disease. Geneva, W.H.O. (Public Health Papers No. 34), 1968.

4 McKeown, T., et al., Screening in Medical Care. London, Oxford University Press, for the Nuffield Provincial Hospitals Trust, 1968.

5 British Medical fournal, 1970, 1, 57.

- Rose, G. A., Health Trends, 1971, 3, 2.

7 Eastwood, M. R., Psychological Medicine, 1971, 1, 197.

8 Kramer, M., in Proceedings of the Third World Congress of Psychiatry. Montreal, McGill University Press, 1961.

- Kendell, R. E., British Fournal of Hospital Medicine, 1971, 6, 147.

10 Shepherd, M., Cooper, B., Brown, A. C., and Kalton, G. W., Psychiatric Illness in General Practice. London, Oxford University Press, 1966.
}

\section{Orbital Varices}

Congenital venous malformations of the orbit are not uncommon. They' nearly always present in childhood or in youth with a proptosis which is aggravated by pressure on the head or neck, and they may be associated with venous dilatations in the neighbouring skin and conjunctiva. Pulsation in such cases normally signifies an arteriovenous shunt, but it may occur in the absence of arteriovenous communication and be attributable to the transmission of cerebral pulsation through an enlarged sphenoidal fissure. Straight $x$-ray examination may provide the diagnosis when the affected orbit is shown to be enlarged, and particularly if there are any concentric rings of calcification from a phlebolith. But the particular value of orbital phlebography in delineating venous malformations within the orbit has been shown in 12 cases of orbital varices described by G. A. S. Lloyd and his colleagues. ${ }^{1}$

Orbital phlebography has been increasingly practised in recent years. It is now usually performed through the frontal rather than the angular vein and after compression over the brow and jugular veins. Compared with carotoid angiography the procedure is relatively simple. It is often done under local anaesthesia and the mortality is nil. As a guide to diagnosis it is valuable not only in frank venous mal- formations but also in many of the other ambiguous disorders presenting merely as symptom-free unilateral exophthalmos.

1 Lloyd, G. A. S., Wright, J. E., and Morgan, G., British Fournal of Ophthalmology, 1971, 55, 505.

\section{Continuous Fetal Heart Rate}

Until recently, because of instrumentation problems, continuous recording of the fetal heart rate was more suited to research than clinical practice. Now with technical improvements the equipment is available for use in the labour ward. The potential advantages of the continuous record to the clinician in terms of the reliable and early detection of fetal asphyxia in labour are considerable. But the record needs to be interpreted differently in certain respects from that of the averaged rate recorded by the midwife when she listens between contractions. The continuous record provides information about the fetal heart rate during uterine contractions, when slowing is common and does not necessarily signify fetal asphyxia.

In 1963 R. Caldeyro-Barcia and co-workers ${ }^{1}$ studied in considerable detail the changes in fetal heart rate of an anencephalic fetus in response to uterine contractions of variable frequency and intensity. They showed that there was a direct relationship between the intensity of the contractions and the amplitude, duration, and timing of the transient fetal bradycardia (dips or decelerations) caused by the contractions. The more intense and frequent the contractions became, the later was the onset of the bradycardia after each contraction, until eventually, when the interval between the point of maximal uterine pressure and of the slowest heart rate exceeded 20 seconds (type II dip in heart rate), the fetus died. This work has frequently been confirmed and forms the basis of all subsequent studies on the evaluation of the continuous fetal heart rate.

Interpretation of changes in the continuous fetal heart rate would be simple if the diagnosis of fetal asphyxia depended on whether type II dips were present or not. E. H. Hon $^{2}$ has shown that asphyxia due to compression of the umbilical cord causes bradycardia early in the contraction phase-a type I dip-and cases have been described in which only type I dips were recorded until shortly before fetal death. ${ }^{3} 4$ Moreover the issue is complicated by the fact that it is uncommon for any two consecutive uterine contractions to produce the same change in heart rate. The amplitude of dips is not necessarily a reflection of the severity of fetal asphyxia, and it is now recognized that shallow type II dips with a slow recovery (late deceleration of $\mathrm{Hon}^{5}$ ) are commonly accompanied by fetal acidosis and a baby born in poor condition. Loss of the normal fluctuations in fetal heart rate, often referred to as "smoothing,"5 or "loss of beat-to-beat variation"6 and tachycardia between contractions are frequent accompaniments of fetal asphyxia.

Attempts have been made to quantitate several features of the continuous fetal heart rate known to be associated with fetal asphyxia into a single expression and relate it to a period of time. ${ }^{78}$ Recently T. Shelley and R. H. Tipton ${ }^{9}$ have combined the independent measurements of frequency, duration, and amplitude of fetal bradycardia by measuring the sum of the products of fall in heart rate over a one-hour period. The figure arrived at is termed the "dip area" and 
has been compared with the Apgar score of the baby one minute after delivery. If the dip area was small, the baby was usually in good condition at birth, but there was no obvious relationship between the dip area and Apgar score of babies in poor condition. The likely reason for this discrepant finding is that the dip area is chiefly a measure of amplitude and does not take into account other changes in fetal heart rate associated with asphyxia such as bradycardia or tachycardia between contractions. ${ }^{10}$ It may be actually misleading when the serious, late, shallow decelerations are the only sign that the fetus is asphyxiated.

In a second paper the authors overcome some of these obiections. ${ }^{11}$ In this they report a composite scoring system, which they have called an index of fetal welfare, for determining the Apgar score of the baby. In addition to the dip area they take account of abnormalities of the fetal heart rate between contractions and meconium staining of the liquor. The index gives remarkably good correlation with the one-minute Apgar score of the baby, but to what extent it is an index of asphyxia is difficult to say. One of the major contributions of these authors has been to draw attention to the importance of meconium staining when the fetal heart rate is abnormal, particularly when the fetus is immature, for in the past this sign has often been overlooked in studies on the detection of fetal asphyxia by continuous heart rate recording. C. J. Hobel ${ }^{12}$ has confirmed this view by showing that in the presence of continuous abnormalities of fetal heart rate acidosis appears earlier when meconium is present.

Any scheme for monitoring the fetus which excludes the use of $\mathrm{pH}$ may be thought incomplete. Heart rate can provide only a limited amount of information about the condition of the asphyxiated fetus, while the $\mathrm{pH}$ is a measure of the metabolic response, which is to some extent independent of circulatory changes. The heart rate is abnormal in about $30 \%$ of high-risk fetuses, but only just under onethird of them actually develop acidosis. ${ }^{4}$ There is also a need at the moment to learn more about the significance of the continuous fetal heart rate in relation to blood gas and acid base values.

All classifications of fetal heart rate patterns such as the index of fetal welfare, owing to their tendency to oversimplify, lead to the loss of useful information. The importance of the studies by Tipton and Shelley is that in constructing their index they have clarified some of the more important signs of fetal asphyxia, but until continuous monitoring is more widely used it would be more informative if these signs were described individually rather than compounding them into a score.

1 Caldeyro-Barcia, R., et al., Modern Problems in Pediatrics, 1963, 8, 267.

2 Hon, E. H., Pan American Health Organization Scientific Publication

3 Wo. 185,1969 , p. 188. of Obstetrics and Gynecology, 1969, 105, 942.

4 Beard, R. W., Brudenell, J. M., Feroze, R. M., and Clayton, S. G., fournal of Obstetrics and Gynaecology of the British Commonwealth, fournal of 78,882 .

${ }^{5}$ Hon, E. H., An Atlas of Fetal Heart Rate Patterns. Connecticut, Harty Press, 1968.

${ }^{6}$ Beard, R. W., Filshie, G. M., Knight, C. A., and Roberts, G. M., fournal of Obstetrics and Gynaecology of the British Commonwealth, 1971, 78,865 .

7 Kubli, R. W., Hon, E. H., Khazin, A. F., and Takemura, H., American fournal of Obstetrics and Gynecology, 1969, 104, 1190.

8 Sureau, C., et al., Gynecologie et Obstetrique, Paris, 1970, 69, 259.

9 Shelley, T., and Tipton, R. H., Fournal of Obstetrics and Gynaecology of the British Commonwealth, 1971, 78, 694 .

10 Coltart, T. M., Trickey, N. R. A., and Beard, R. W., British Medical fournal, 1969, 1, 342.

11 Tipton, R. H., and Shelley, T., Fournal of Obstetrics and Gynaecology of the British Commonwealth, 1971, 78, 702 .

12 Hobel, C. J., American Fournal of Obstetrics and Gynecology, 1971, 110, 336 .

\section{Clofibrate in Ischaemic Heart Disease}

Ischaemic heart disease is the cause of more than a quarter of all deaths in the United Kingdom, and epidemiological studies in many countries have shown its incidence is increased in those who are overweight, inactive, smoke, or have high serum lipid levels. Not surprisingly, in recent years increasing attention has been given to measures which might protect the patient who already has ischaemic heart disease in the community-primary protection-or which might protect the patient who already has ischaemic heart disease from sudden death or myocardial infarctionsecondary prevention.

Among the measures proposed for primary prevention of ischaemic heart disease have been reduction in weight, increase in physical exercise, stopping smoking, and adoption of special diets. All these measures are easy to propose but difficult for most citizens to accept. But when a patient has already had his first myocardial infarction or is suffering from angina, though it is late in the day, he is more ready to listen to unpalatable advice and is prepared also to accept long-continued drug treatment.

Unfortunately the natural history of i.chaemic heart disease is such that it is not easy to measure the effectiveness of such measures. The one enduring legacy of the unhappy experience of the use of anticoagulant therapy is an appreciation of the importance of carefully planned and properly controlled therapeutic trials. The enthusiasm generated by the early trials of anticoagulants, trials which in retrospect were poorly planned and inadequately controlled, 1 created a belief that failure to use anticoagulants in patients who had had a myocardial infarct almost constituted negligence. This impeded further studies and resulted in many patients receiving treatment which was not without hazard and which was later shown to be of little value. ${ }^{2}$

After the anticoagulants, interest turned to drugs which reduce serum lipid levels, such as oestrogens, triparanol (MER 29), nicotinic acid, and clofibrate (Atromid S). The situation five years ago was that clofibrate looked the most promising, but evidence was needed whether the use of this drug to reduce serum lipid levels benefited patients. Now in this issue of the B.M.F. two large groups of physicians in Scotland and Newcastle upon Tyne report wellplanned, controlled trials of clofibrate in which they have watched 1,214 patients for four or five years (pp. 775 and 767). Dr. M. F. Oliver in Edinburgh and Dr. H. A. Dewar in Newcastle, who undertook the planning of these trials, have prepared a joint commentary (p. 784) on the reports. The results of the trials are in some ways disappointing. Of 620 patients in the two trials who did not receive clofibrate, $79(13 \%)$ died suddenly from myocardial infarction during the period of observation, while of 594 patients who did receive clofibrate $59(10 \%)$ died. This bare statement suggests that this therapy, like anticoagulant therapy 15 years ago, is not going to make much impact on ischaemic heart disease. But detailed analysis reveals important, but puzzling results. Patients with pre-existing angina were protected more by clofibrate than patients who had suffered myocardial infarction but had not had angina, and the protection seemed unrelated to the effect of clofibrate on serum lipids. This protection was shown in a reduction in the number of both fatal and non-fatal infarcts in the treated groups. But the differences between the findings in 